\title{
Anatomie des distalen Radius
}

\author{
Maika Szermutzky, Johannes Frank, Ingo Marzi
}

\section{Zusammenfassung}

Der distale Radius ist die Hauptkomponente im Handgelenk und für die Kraftübertragung verantwortlich. Die knöchernen Strukturen und Bandverbindungen bilden dabei die Grundlage für die hohe Beweglichkeit und für die Kraftübertragung vom Unterarm auf die Hand. Das Handgelenk setzt sich dabei proximal aus der radiokarpalen $\mathrm{Ge}$ lenkfläche, dem distalen Radioulnargelenk und dem triangulären Faserknorpel (TFC) zusammen. Distal wird das Handgelenk von der ersten Handwurzelknochenreihe gebildet, die durch intrinsische Bänder zusammengehalten wird. Die Stabilität des Handgelenks wird durch die Gelenkkapsel und v.a. durch die überbrückenden dorsalen und palmaren extrinsischen Bänder gebildet. Verletzungen des distalen Radius mit Verkürzung und Verkippung führen regelmäßig zu einer Mitverlet- zung dieser Strukturen und damit zu funktionellen Defiziten. Diese Verletzungen erfordern häufig eine differenzierte klinische, radiologische oder arthroskopische Diagnostik. Neben einer möglichst anatomischen Wiederherstellung des distalen Radius müssen daher die Verletzungen des distalen Radioulnargelenks (DRUG), des TFC und der wichtigen Bandstrukturen beachtet werden. Das funktionelle Ergebnis ergibt sich daher aus der anatomischen Wiederherstellung des distalen Radius einschließlich seiner unmittelbaren Begleitstrukturen.

\section{Anatomy of the Distal Radius}

The distal radius is the main component of the wrist and responsible for power transmission. The basis for high mobility and power transmission from forearm to the hand is provided by the bony structures and ligament connec- tions. The wrist consists proximal of the radial-carpal joint, the distal radioulnar joint and the triangular fibrocartilage (TFC). Distally it is formed by the first row of carpal bones, which are tightly connected by intrinsic ligaments. The stability of the wrist is formed by the joint capsule, and especially by bridging of the dorsal and palmar extrinsic ligaments. An injury to the distal radius with shortening and angulation regularly leads to an injury of these structures and thereby to functional deficits. These injuries often necessitate a differentiated clinical, radiological or arthroscopic diagnostic process. In addition to a nearly anatomic restoration of the distal radius, therefore, the injuries of the DRUJ, the TFC and the major ligamentous structures, need to be addressed. The functional outcome is related to the anatomic restoration of the distal radius including its directly neighbouring structures.

\section{Einleitung}

Der distale Radius stellt den Hauptpfeiler des Handgelenks dar und ist für die Kraftübertragung verantwortlich. Die radiokarpale Gelenkfläche wird nach ulnar hin durch den triangularen Faserkomplex (TFC) vervollständigt und ist mit dem distalen Radioulnargelenk (DRUG) eng verbunden. Die Verbindung zur Hand selbst erfolgt über starke dorsale und palmare Bandverbindungen $\mathrm{zu}$ den Handwurzelknochen.

Diese komplexe anatomische Konstellation erlaubt sowohl einen hohen Bewegungsgrad im Handgelenk als auch die Unterarmrotation. Dieser Aufbau führt

OP-JOURNAL 2012; 28: 224-227

(c) Georg Thieme Verlag KG Stuttgart · New York DOI http://dx.doi.org/10.1055/s-0032-1327996 jedoch dazu, dass bei Verletzungen des distalen Radius diese anatomischen Strukturen immer mitbeurteilt und therapiert werden müssen (Abb. 1).

\section{Hauptteil}

Das proximale Handgelenk wird gebildet von der Facies articularis carpalis des Radius und dem Discus articularis, welcher dem Ulnakopf aufliegt. Zusammen bilden sie die Gelenkpfanne. Die proximale Reihe der Handwurzelknochen bildet dabei den Gelenkkopf.

Dieses so gebildete Gelenk ist ein Ellipsoidgelenk, welches ausgesprochen beweglich ist und 2 Freiheitsgrade ermöglicht: Extension/Flexion (bis $120^{\circ}$ ) sowie Ulnar-/Radialabduktion (bis $50^{\circ}$ ).

Die Facies articularis carpalis wird durch 2 konkave Gelenkflächen gebildet: die
Fossa scaphoidea und die Fossa lunata. Die Fossa scaphoidea hat eine Dreiecksform, dessen Spitze den Processus styloideus radii formt. Eine dorsopalmare Crista trennt die Fossa scaphoidea von der kleineren Fossa lunata. Beide Gelenkflächen sind konkav in der anterior/ posterioren und radial/ulnaren Ebene.

Zudem hat der Radius noch eine 3. konkave Gelenkfläche: die Fossa der Articulatio radioulnaris distalis, welche auch als sog. „Sigmoid Notch“ bezeichnet wird (Abb. 2). Mit dieser Gelenkfläche artikuliert der Radius mit der Circumferentia articularis des Caput ulnae. Die an der Knorpelknochengrenze befestigte Gelenkkapsel erweitert sich dort zu einem Recessus sacciformis distalis, wodurch der notwendige Bewegungsspielraum für Drehbewegungen ermöglicht wird. Die Fossa der Articulatio radioulnaris distalis bildet somit die konkave Gelenk- 


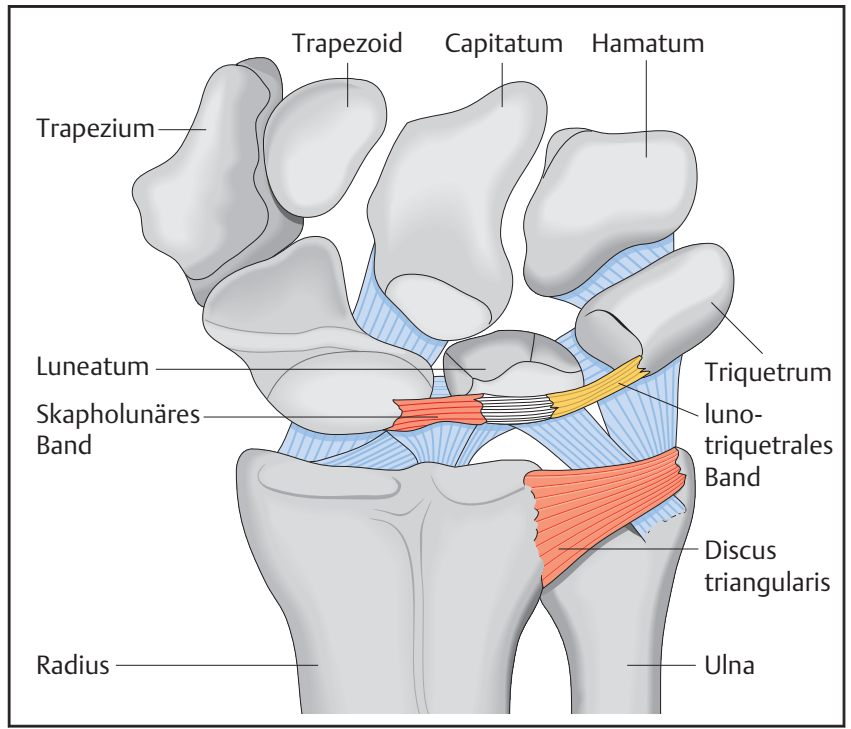

Abb. 1 Schematische Darstellung des Handgelenks mit distaler Ulna und Radius sowie die angrenzenden Handwurzelknochen und die wichtigsten Begleitstrukturen.

fläche des distalen Radioulnargelenks (DRUG) und ist nach distal, dorsal und palmar scharf begrenzt, nicht jedoch nach proximal.

Zwischen dem Processus styloideus ulnae und der ulnaren Seite des Radius befindet sich der faserknorpelige Discus articularis (TFC bzw. Discus triangularis). Der TFC inseriert an der distalen ulnarseitigen Gelenkfläche des Radius und zieht über die distale Ulna sowohl zum Processus styloideus ulnae als auch der Fovea des Ulnakopfes. Er dient als Gelenkscheibe zum Os triquetrum und Os lunatum sowie als Verbindung zwischen Ulna und Radius. Zudem vervollständigt er die Gelenkfläche ulnarseitig. Weiterhin hat die Ulna durch den TFC keinen direkten Kontakt zur proximalen Handwurzelreihe.

Durch die gelenkige Verbindung zwischen Radius und Ulna kann eine Drehbewegung um die Ulna (Pro- und Supination) ermöglicht werden. Dabei erfolgt die Rotation im distalen Radioulnargelenk durch eine Rotation des Radius mit der Hand um den Ulnakopf, welcher durch die Ulna und die Struktur des Ellenbogengelenks fixiert ist. Es kann eine Rotation bis etwa $150^{\circ}$ erreicht werden.

Um einerseits eine uneingeschränkte Unterarmrotation im distalen Radioulnargelenk (DRUG) zu ermöglichen und andererseits die Ulnarabduktion sowie eine freie Bewegung im Handgelenk zu gewährleisten, ist eine korrekte Länge und Stellung der distalen Ulna und des distalen Radius von erheblicher Bedeutung.

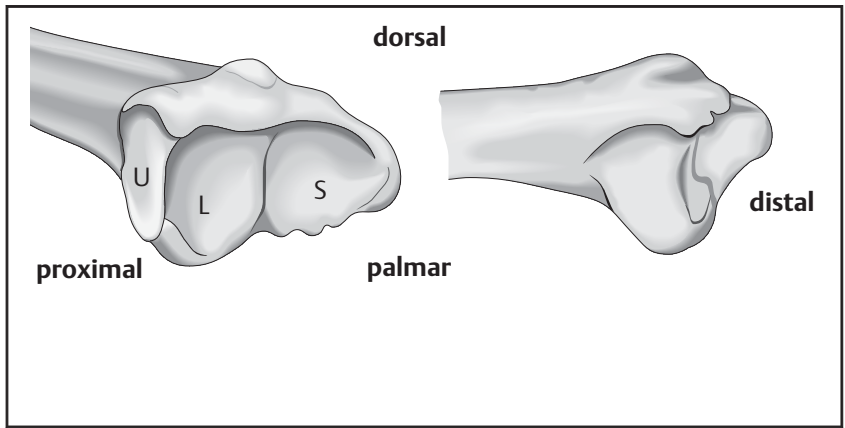

Abb. 2 Darstellung der Anatomie des distalen Radius mit der Articulatio radioulnaris distalis (,Sigmoid Notch“ $=\mathrm{U}$ ) sowie der Fascies articularis carpalis, welche aus der Fossa scaphoidea (=S) und der Fossa lunata $(=\mathrm{L})$ besteht.

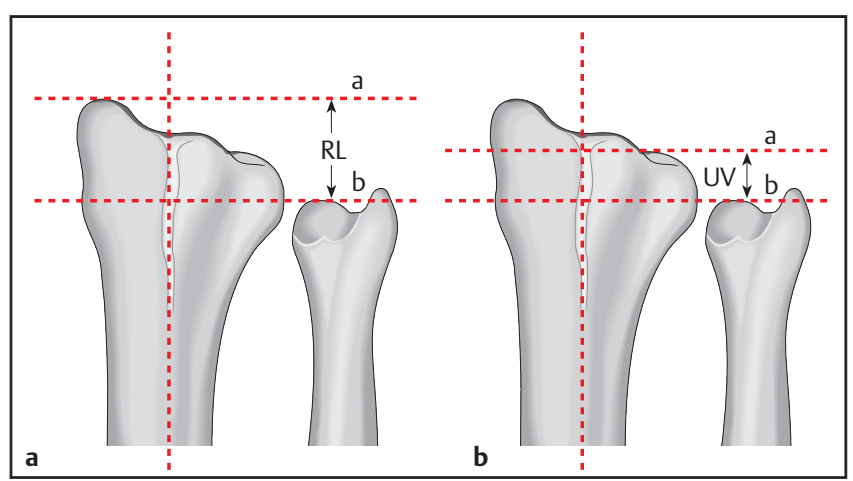

Abb. $\mathbf{3} \mathbf{a}$ und $\mathbf{b}$ Schematische Darstellung zur Bestimmung der radialen Länge am distalen Radius und der Ulnavarianz. a Die radiale Länge wird im a.-p.-Strahlengang bestimmt und ist der Abstand zwischen der Senkrechten zur Achse des Radius durch die Spitze des Processus styloideus radii (a) und der Senkrechten in Höhe der distalen Gelenkfläche des Ulnakopfes (b). Im Normalfall beträgt die Länge ca. $12 \mathrm{~mm}$ (8$18 \mathrm{~mm}$ ). b Die Ulnavarianz wird ermittelt aus dem Abstand zwischen der Parallelen zur Fossa lunata (a) und der zur distalen Gelenkfläche des Ulnakopfes (b). Bei ca. $60 \%$ befindet sich die distale Gelenkfläche des Ulnakopfes und der ulnare Rand des Radius auf der gleichen Höhe. Es findet sich eine Variation zwischen +4 und $-4 \mathrm{~mm}$ ohne Pathologie.

Die individuelle Längenrelation von distaler Ulna und Radius unterliegt einer Varianz, die bei der Beurteilung gegebenenfalls einen Vergleich mit der Gegenseite erfordert. Man geht davon aus, dass Variationen bis zu $4 \mathrm{~mm}$ ohne pathologische Bedeutung sind. Die korrekte Erfassung der Ulnavarianz ist in Abb. 3 dargestellt $[1,2,7]$.

Der metaphysäre Bereich des Radius beginnt etwa $2 \mathrm{~cm}$ vor der distalen Gelenkfläche und ist gekennzeichnet durch eine Abnahme der Dicke der Kortikalis bei gleichzeitiger Zunahme an spongiösem Knochen. In der frontalen Ebene ist die Radiusgelenkfläche mit einem Winkel von ca. $22-23^{\circ}\left(13-30^{\circ}\right)$ nach ulnar geneigt und stellt den knöchernen tragenden Anteil des Handgelenksbogens dar (Ulnarinklination) (Abb.4). In der sagittalen Ebene ist die Gelenkfläche des Ra- dius im Durchschnitt zwischen 11-12 $\left(0-28^{\circ}\right)$ nach palmar geneigt (Palmarinklination) (Abb. 5).

Diese Inklination ist für die korrekte Kraftübertragung zwischen dem distalen Radius und den Handwurzelknochen von erheblicher Bedeutung.

Der palmare Bereich des Radius ist flach und stellt den Ansatzpunkt für wichtige, das Handgelenk stabilisierende Bandstrukturen dar. Der dorsale Bereich hat eher eine konvexe Form und dient als Widerlager für die 6 dorsalen Strecksehnenfächer. Im Bereich des Processus styloideus radii findet sich manchmal eine Rinne für die Sehnen des 1. Strecksehnenfachs. Zusätzlich findet sich eine längliche Erhöhung, das Tuberculum Lister (Tuberculum dorsale), welches der Sehne des 3. Strecksehnenfachs, dem 


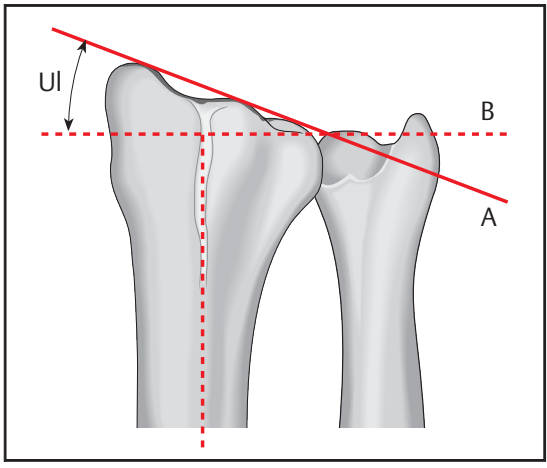

Abb. 4 Bestimmung der Ulnarinklination des distalen Radius. Die Ulnarinklination wird bestimmt als Winkel (UI) zwischen der Paralelen zur Gelenkfläche (A) und der Senkrechten zur Längsachse des Radius (B). Im Normalfall beträgt dieser ca. $23^{\circ}\left(13-30^{\circ}\right)$.

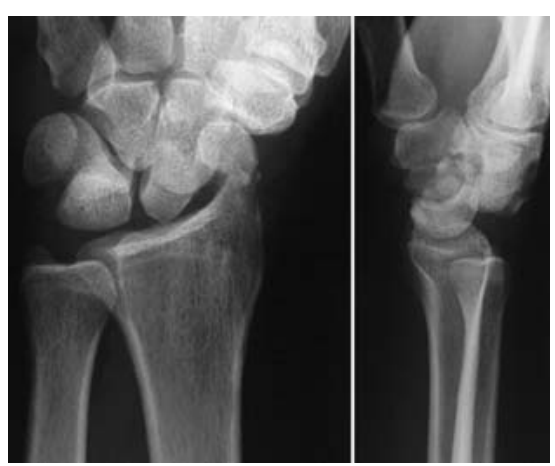

Abb. 7 Dieses Röntgenbild zeigt eine nach transscaphoidär perilunäre Luxationsfraktur. Die Bögen sind alle unterbrochen und im seitlichen Strahlengang ist deutlich die Luxationsstellung zwischen Lunatum und Capitatum zu erkennen.

langen Daumenstrecker, als Widerlager dient. Diese Sehne des M. extensor pollicis longus umläuft diese Knochenstruktur, die ihm als Hypomochlion dient.

Untersuchungen haben gezeigt, dass etwa $80 \%$ der axialen Belastung über die

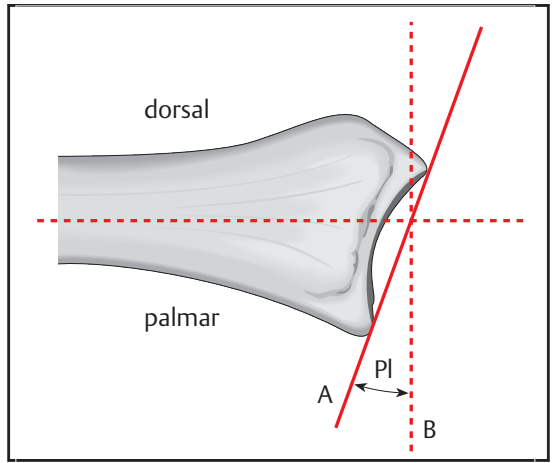

Abb. 5 Bestimmung der Palmarinklination des distalen Radius. Die Palmarinklination wird bestimmt als Winkel (PI) zwischen der Parallelen zur Gelenkfläche (A) und der Senkrechten zur Längsachse des Radius (B). Im Normalfall beträgt diese ca. $12^{\circ}\left(0-28^{\circ}\right)$.

radiokarpale Gelenkfläche auf den Unterarm übertragen wird. $20 \%$ werden über das ulnare Kompartment auf die 1. Handwurzelknochenreihe weitergeleitet. Damit diese Kräfte übertragen werden können und dennoch der hohe Grad an Beweglichkeit bewahrt werden kann, sind komplexe Band- und Weichteilstrukturen zur Sicherung der Stabilität erforderlich. Die Integrität und Stabilität der Bandstrukturen lässt sich am Standardröntgenbild nur indirekt beurteilen.

Als gute Orientierung hat sich hier die Verfolgung dreier von Gilula beschriebenen bogenförmigen Linien bewährt. Diese Linien sollen im Normalfall ohne Versatz oder Unterbrechung den Verlauf der Gelenkflächen der Handwurzelknochen und der karpalen Gelenkflächen als rundliche Bögen nachziehen und verlaufen parallel (Abb. 6).

Bei einer Luxation oder Luxationsfraktur sind diese Bögen unterbrochen und weisen auf eine schwerwiegende Verletzung hin (Abb. 7) [5, 7].

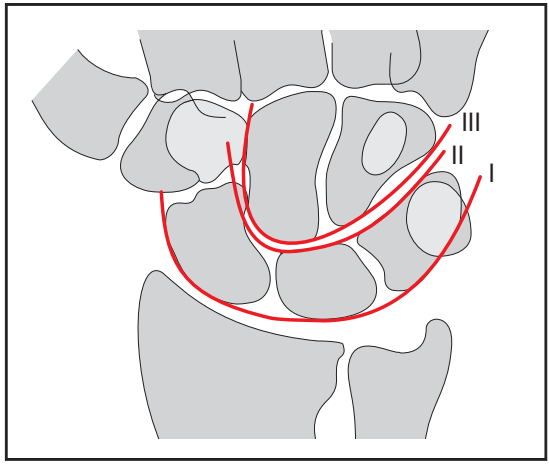

Abb. 6 Hilfreich für die Beurteilung der Stellung der Handwurzelknochen sind die 3 von Gulila beschriebenen Bögen. Diese „karpalen“ Bögen sollten im Normalfall glatt entlang der Gelenkflächen der Handwurzelknochen verlaufen ohne plötzlichen Sprung oder unterschiedliche Weiten der Gelenkspalten (Bogen I, II und III) (nach Schmitt R, Lanz U. Bildgebende Diagnostik der Hand. Stuttgart: Thieme; 1996: 251).

Die Stabilität des Handgelenks wird von extrinsischen und intrinsischen Bändern gegeben. Diese ziehen vom Radius und der Ulna zu den Handwurzelknochen der 1. und 2. Reihe (s. Abb. 1). Die extrinsischen Bänder überbrücken palmar und dorsal das Radiokarpal-, Ulnokarpaloder das Interkarpalgelenk (Abb. 8). Radiokarpal finden sich hier auf der Palmarseite das radioskaphokapitäre Band, das lange und kurze radiolunäre Band und das radioskapholunäre Band. Auf der ulnaren palmaren Seite sind hier insbesondere das ulnokapitäre, ulnotriquetrale und ulnolunäre Band $\mathrm{zu}$ nennen, die den TFC stabilisieren. Die intrinsischen Bänder verbinden hingegen die einzelnen Handwurzelknochen miteinander, wie das skapholunäre und das lunotriquetrale Band (Abb. 9). Dorsalseitig wird das Radiokarpalgelenk durch das radiotriquetrale Band stabilisiert.

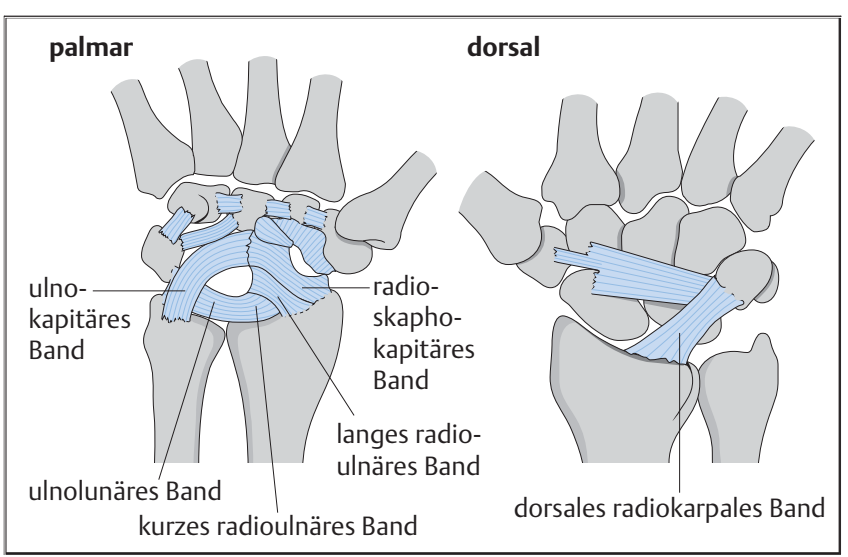

Abb. 8 Schemazeichung der extrinsischen karpalen Bänder.

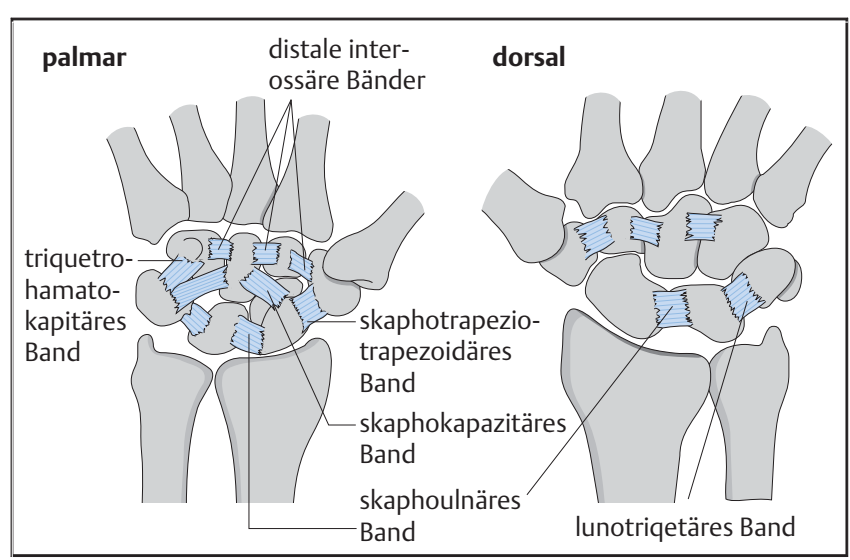

Abb.9 Schemazeichung der intrinsischen karpalen Bänder. 
Tab. 1 Karpale Bandstrukturen.

\section{extrinsische Bänder}

oberflächliche und tiefe radiokarpale Bänder

- radioskaphokapitäres Ligament (RSC)

- langes radiolunäres Ligament (LRL)

- radioskapholunäres Ligament (RSL)

- kurzes radiolunäres Ligament (SRL)

- dorsales radiotriquetrales Ligament (RTq)

oberflächliche und tiefe ulnokarpale Bänder

- ulnokapitäres Ligament (UC)

- ulnotriquetrales Ligament (UTq)

- ulnolunäres Ligament (UL)

\begin{tabular}{ll}
\hline distale Reihe:interossäre Bänder \\
\hline palmare interkarpale Bänder \\
- triquetrohämatoskaphitäres Ligament \\
(TqHC) \\
- skaphokapitäres Ligament \\
- skaphotrapeziotrapezoidäres Ligament \\
(STT) \\
dorsale interkarpale Bänder (DIC)
\end{tabular}

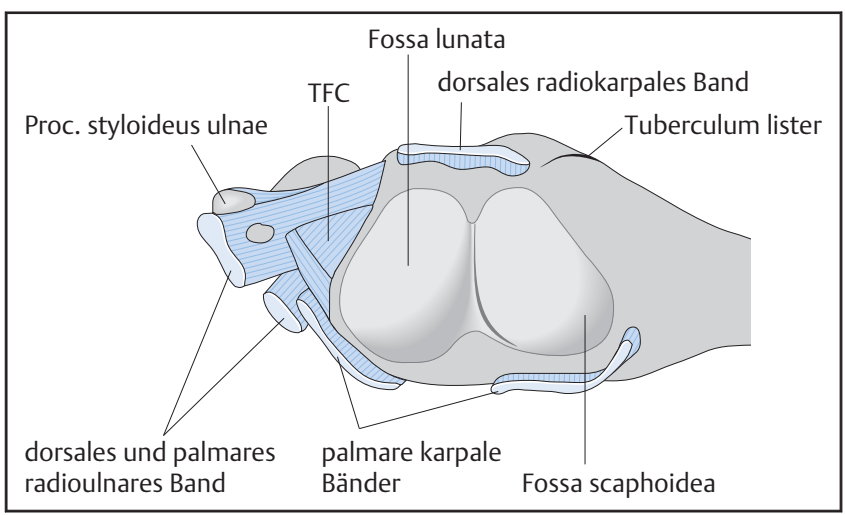

Abb. 10 Schematische Darstellung des TFC bzw. des distalen Radioulnargelenks (DRUG) mit den entsprechenden stabilisierenden Bandstrukturen.

Eine Übersicht über die relevanten Bandstrukturen gibt Tab. 1 [4-7].

Von erheblicher Bedeutung sind die Rupturen des skapholunären (SL) Bandes, die insbesondere bei B1- und CFrakturen des Radius beobachtet werden.

Ein Übersehen einer Verletzung dieser Bandstrukturen kann zu einem zunehmenden Kollaps der proximalen Handwurzelreihe mit erheblicher Schmerzhaftigkeit und Bewegungseinschränkung bis hin zur Arthrose führen, weshalb dem Erkennen und Wiederherstellen der Strukturen eine erhebliche Bedeutung zukommt [3].

Die Fossa articularis carpalis wird also durch palmare und dorsale Bandstruktu- ren überbrückt. Die extrinsischen Bandstrukturen umgreifen jedoch auch das ulnare Kompartment mit dem Discus triangularis (TFC) im Zentrum. Der dorsale und palmare Anteil werden als dorsales und palmares radioulnares Band bezeichnet und stabilisieren das distale Radioulnargelenk bei den Umwendbewegungen des Unterarms (Abb. 10). Die Gesamtheit aus diesen Bändern und dem TFC werden als TFCC (triangular fibrocartilage complex) bezeichnet und stabilisiert neben dem distalen Radioulnargelenk das ulnare Handgelenk. Das distale Radioulnargelenk (DRUG) wird zusätzlich durch folgende Faktoren stabilisiert: die interossäre Membran zwischen Radius und Ulna, den M. pronator quadratus, die Sehnen und Sehnenscheiden des M. extensor carpi ulnaris und des M. flexor carpi ulnaris.
Posttraumatische Fehlstellungen des distalen Radius mit relativer Verschiebung zur Ulna hin können zu einer schmerzhaften posttraumatischen Arthrose im DRUG führen.

\section{Schlussfolgerung}

Bei allen Verletzungen des distalen Radius müssen immer die eng verbundenen anatomischen Strukturen und funktionellen Abläufe berücksichtigt werden. Eine möglichst exakte Wiederherstellung der Länge, Achse und Neigung des distalen Radius ist Voraussetzung für eine optimale Funktion des Handgelenks. Dabei sind das Erkennen und die Mitbehandlung der möglichen Begleitverletzungen für das subjektive und objektive Behandlungsergebnis distaler Radiusfrakturen von entscheidender Bedeutung.

\section{Literatur}

${ }^{1}$ Aro HT, Koivunen T. Minor axial shortening of the radius affects outcome of Colles' fracture treatment. J Hand Surg Am 1991; 16: 392 398

${ }^{2}$ Fernandez DL, Jupiter JB. Fractures of the distal Radius. A practical Approach to Management. New York, Berlin, Heidelberg: Springer; 1996

3 Frank J, Pralle H, Marzi I. Hand und Finger. In: Scharf H-P, Rüter A, Pohlemann T et al., Hrsg. Orthopädie und Unfallchirurgie. München: Elsevier Urban und Fischer; 2011: 557-593

4 Frank J, Marzi I. Handchirurgie. In: Bruch H-P, Trentz O. Chirurgie. München: Elsevier Urban und Fischer; 2008: 507-545

5 Garcia-Elias M. Kinetic analysis of carpal stability during grip. Hand Clin 1997; 13: 151158

6 Geissler WB, Freeland AE, Savoie FH et al. Whipple: Intracarpal soft-tissue lesions associated with an intra-articular fracture of the distal end of the radius. J Bone Joint Surg [Am] 1996; 78: 357-365

7 Green DP, Hotchkiss RN, Pederson WC. Greenys operative Hand Surgery. New York, Edinburgh, London, Philadelphia, San Francisco: Churchill Livingstone; 1999

\section{Dr. med. Maika Szermutzky}

Assistenzärztin

Prof. Dr. med. Johannes Frank

Oberarzt

Prof. Dr. med. Ingo Marzi

Chefarzt

Klinik für Unfall-, Hand- und

Wiederherstellungschirurgie

Uniklinik Frankfurt am Main

Theodor-Stern-Kai 7

60590 Frankfurt am Main

Maika.Szermutzky@kgu.de 